\title{
RESEARCH
}

Open Access

\section{In vivo imaging of sterile microglial activation in rat brain after disrupting the blood-brain barrier with pulsed focused ultrasound: [18F]DPA-714 PET study}

Sanhita Sinharay ${ }^{1,6}$, Tsang-Wei Tu ${ }^{2,3,7}$, Zsofia I. Kovacs ${ }^{2,8}$, William Schreiber-Stainthorp ${ }^{1}$, Maggie Sundby ${ }^{2}$, Xiang Zhang ${ }^{4}$, Georgios Z. Papadakis ${ }^{1,9}$, William C. Reid ${ }^{1}$, Joseph A. Frank ${ }^{2,5}$ and Dima A. Hammoud ${ }^{1 *}$ (D)

\begin{abstract}
Background: Magnetic resonance imaging (MRI)-guided pulsed focused ultrasound combined with the infusion of microbubbles ( $\mathrm{pFUS}+\mathrm{MB}$ ) induces transient blood-brain barrier opening (BBBO) in targeted regions. pFUS+MB, through the facilitation of neurotherapeutics' delivery, has been advocated as an adjuvant treatment for neurodegenerative diseases and malignancies. Sterile neuroinflammation has been recently described following pFUS+MB BBBO. In this study, we used PET imaging with [18F]-DPA714, a biomarker of translocator protein (TSPO), to assess for neuroinflammatory changes following single and multiple pFUS+MB sessions.

Methods: Three groups of Sprague-Dawley female rats received MRI-guided pFUS+MB (Optison ${ }^{\mathrm{TM}} ; 5-8 \times$ $10^{7} \mathrm{MB} / \mathrm{rat}$ ) treatments to the left frontal cortex and right hippocampus. Group A rats were sonicated once. Group $B$ rats were sonicated twice and group $C$ rats were sonicated six times on weekly basis. Passive cavitation detection feedback (PCD) controlled the peak negative pressure during sonication. We performed T1-weighted scans immediately after sonication to assess efficiency of BBBO and T2*-weighted scans to evaluate for hypointense voxels. [18F]DPA-714 PET/CT scans were acquired after the BBB had closed, $24 \mathrm{~h}$ after sonication in group $A$ and within an average of 10 days from the last sonication in groups $B$ and $C$. Ratios of T1 enhancement, T2* values, and [18F]DPA-714 percent injected dose/cc (\%ID/cc) values in the targeted areas to the contralateral brain were calculated. Histological assessment for microglial activation/ astrocytosis was performed.
\end{abstract}

Results: In all groups, [18F]DPA-714 binding was increased at the sonicated compared to non-sonicated brain (\%ID/cc ratios $>1)$. Immunohistopathology showed increased staining for microglial and astrocytic markers in the sonicated frontal cortex compared to contralateral brain and to a lesser extent in the sonicated hippocampus. Using MRI, we documented BBB disruption immediately after sonication with resolution of BBBO $24 \mathrm{~h}$ later. We found more T2* hypointense voxels with increasing number of sonications. In a longitudinal group of animals imaged after two and after six sonications, there was no cumulative increase of neuroinflammation on PET.

(Continued on next page)

\footnotetext{
* Correspondence: hammoudd@cc.nih.gov

${ }^{1}$ Hammoud Laboratory, Center for Infectious Disease Imaging, Clinical Center,

National Institutes of Health, 10 Center Drive, Building 10, Room 1C-368,

Bethesda, MD 20892, USA

Full list of author information is available at the end of the article
}

(c) The Author(s). 2019 Open Access This article is distributed under the terms of the Creative Commons Attribution 4.0 International License (http://creativecommons.org/licenses/by/4.0/), which permits unrestricted use, distribution, and

reproduction in any medium, provided you give appropriate credit to the original author(s) and the source, provide a link to the Creative Commons license, and indicate if changes were made. The Creative Commons Public Domain Dedication waiver (http://creativecommons.org/publicdomain/zero/1.0/) applies to the data made available in this article, unless otherwise stated. 
(Continued from previous page)

Conclusion: Using [18F]DPA-714 PET, we documented in vivo neuroinflammatory changes in association with pFUS+MB. Our protocol (utilizing PCD feedback to minimize damage) resulted in neuroinflammation visualized $24 \mathrm{~h}$ post one sonication. Our findings were supported by immunohistochemistry showing microglial activation and astrocytosis. Experimental sonication parameters intended for BBB disruption should be evaluated for neuroinflammatory sequelae prior to implementation in clinical trials.

Keywords: Neuroinflammation, [18F]DPA-714 PET, Pulsed focused ultrasound, Magnetic resonance imaging, Translocator protein

\section{Introduction}

The highly selective nature of the blood-brain barrier (BBB) limits the effective delivery of neurotherapeutics to the brain. The BBB consists of a combination of specialized endothelial cells, junctional complexes between adjacent endothelial cells, basement membranes, and perivascular structures comprising the neurovascular unit (NVU) [1, 2]. Several strategies have so far been employed to effectively bypass the BBB for enhanced localized delivery of drugs, genes, antibodies, and nanoparticles [3-7]. Among these techniques, noninvasive MRI-guided pulsed focused ultrasound with intravenous injection of microbubbles ( $\mathrm{pFUS}+\mathrm{MB}$ ) has emerged as a promising approach for targeted transient $\mathrm{BBB}$ opening (BBBO) [8-12].

Although the exact mechanism of BBBO is still unclear, it has been postulated that pFUS+MB generates acoustic radiation forces in association with the intravascular MB which results in oscillations leading to the propagation of pressure waves and interaction with and through the NVU endothelium, resulting in decreased tight junction integrity [13, 14]. This process has long been assumed to be benign in nature with minimal damage. More recently, however, a rapid increase in damage-associated molecular pattern (DAMP) factors [15-17] was observed following pFUS+MB, with increased expression of heat shock protein 70 (HSP70) and proinflammatory cytokines including tumor necrosis factor alpha (TNF $\alpha$ ), interleukin (IL) $1 \alpha$, IL1 $\beta$, IL18, and interferon gamma (IFN $\gamma$ ) in sonicated animal brains $[14$, 18], suggesting a neuroinflammatory process.

MR imaging with gadolinium chelate contrast administration has been used extensively in the evaluation of pFUS +MB BBBO in animal models. The efficacy of BBBO post pFUS $+\mathrm{MB}$ is generally demonstrated on contrastenhanced T1-weighted images in animal models and clinically as areas of contrast extravasation [12, 19-22]. The MRI changes detected following sonication include T2 hyperintensities, microhemorrhages, and enlarged ventricles [14, 23, 24]. However, MRI cannot directly visualize inflammatory changes associated with pFUS+MB treatments. On the other hand, positron emission tomography (PET) imaging can assess neuroinflammation, non-invasively, through targeting of the translocator protein (TSPO), an outer mitochondrial membrane receptor known to be upregulated in activated microglia and macrophages $[25,26]$. One TSPO ligand, ${ }^{18} \mathrm{~F}$ DPA714, has already shown promise in detecting microglial activation in preclinical disease models [27-29] as well as in patients with Alzheimer's disease $(\mathrm{AD})$ and stroke [30, 31].

In this study, we used [18F]DPA-714 as an in vivo biomarker of neuroinflammation in pFUS+MB-treated rats. First, we probed for effective BBBO immediately after sonication with MRI and assessed for resolution of BBBO $24 \mathrm{~h}$ after sonication. We compared [18F]DPA714 binding in the sonicated left frontal cortical and right hippocampal regions to the contralateral intact brain after one, two, and six sonications. We evaluated the potential cumulative effect of multiple sonications on the inflammatory response by longitudinally evaluating a subgroup of rats after receiving two and six weekly pFUS +MB treatments. Finally, we confirmed our PET findings with immunofluorescent staining for microglial activation and astrocytosis.

\section{Methods}

\section{Animals}

All experiments were approved by the Animal Care and Use Committee (ACUC) of the Clinical Center (CC) at the National Institutes of Health (NIH). Sprague-Dawley female rats (8-10 week old, $n=21)$ were used for all experiments (Charles River Laboratory, Wilmington, MA). Animals were housed in the small animal housing facility at an ambient temperature of $72 \pm 2{ }^{\circ} \mathrm{F}\left(21-23{ }^{\circ} \mathrm{C}\right)$ which is within the range suggested by the national research Council [32]. Animals were allowed free access to food and water, with a $12 \mathrm{~h}$ light/dark cycle. All MR and PET imaging experiments were performed within the light cycle. The animals were kept warm during various procedures using heating pads.

The animals were divided into three groups. Group A rats $(n=6)$ received one pFUS $+\mathrm{MB}$ treatment and were PET imaged $24 \mathrm{~h}$ after sonication. Group B rats $(n=5)$ received two weekly sonications $(2 \times)$ and underwent PET 13-14 days after the second sonication. Finally, 


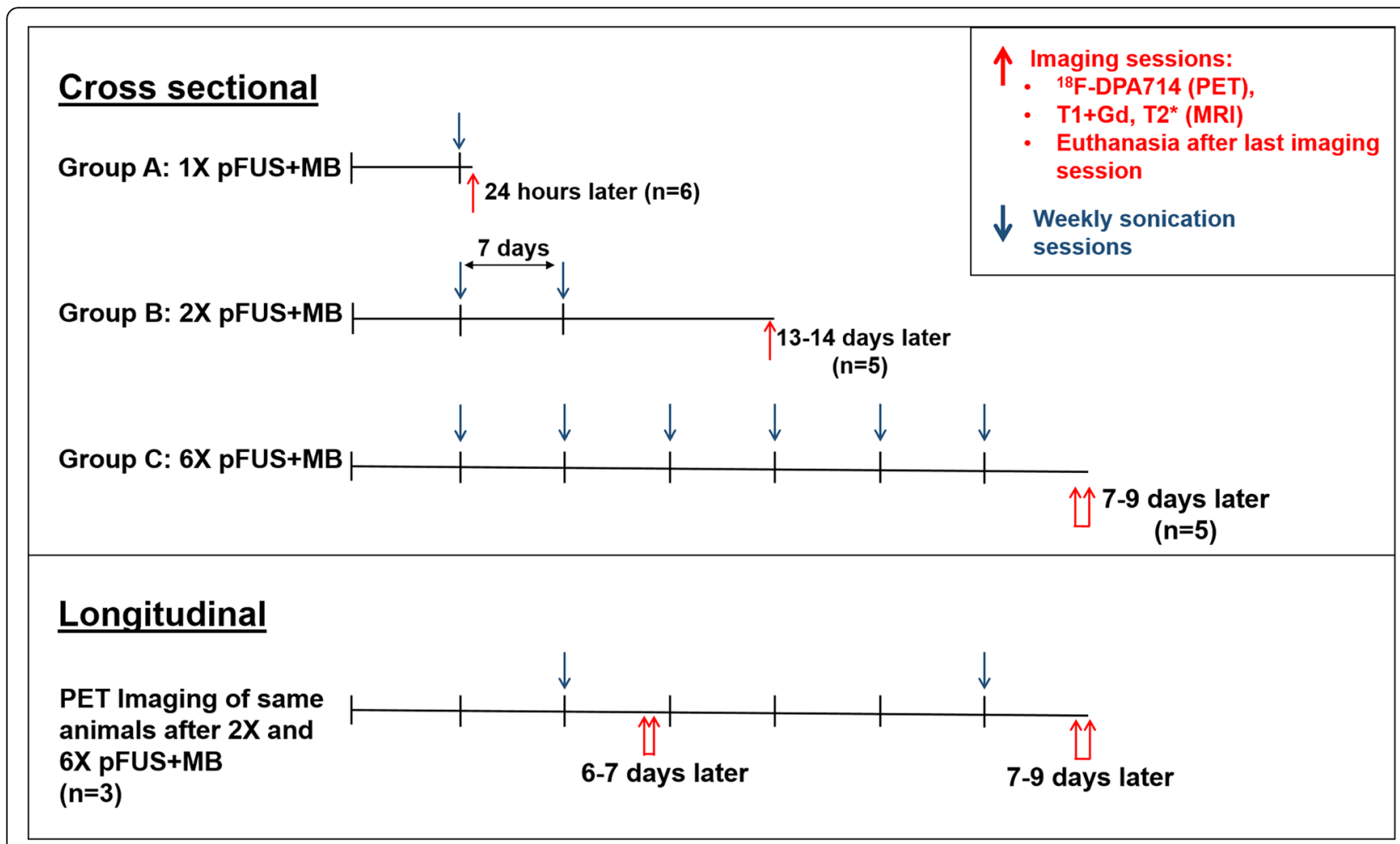

Fig. 1 Schematic representation of the pFUS+MB, PET, and MR imaging sessions for cross-sectional and longitudinal components

group $C$ rats $(n=5)$ received six weekly sonications $(6 \times)$ and were imaged 7-9 days after the last sonication session.

Three animals from group C (six sonications) were also imaged after two sonications and as such were used for longitudinal imaging $(n=3$, PET imaging performed 5-6 days after the second sonication and 7-9 days after the sixth sonication). The imaging data from the second sonication in the longitudinal group was not used for the cross-sectional analysis. All animals were euthanized following their last PET imaging session (Fig. 1).

In a separate experiment to document reversal of $\mathrm{BBBO}$ after sonication, we performed $\mathrm{MR}$ imaging on five rats immediately after sonication as well as $24 \mathrm{~h}$ later, using pre and post contrast T1-weighted images.

\section{MRI-guided pFUS+MB and MR imaging (in vivo and ex vivo)}

Rats were first anesthetized with isoflurane $(1-3.5 \%)$ on $100 \% \mathrm{O}_{2}$ as previously described [14, 24]. To determine the pFUS targeting coordinates in the left frontal cortex and right hippocampal regions, axial turbo-spin echo (TSE) T2-weighted images with repetition time/echo time $(\mathrm{TR} / \mathrm{TE}=2000 / 60 \mathrm{~ms})$ of the rat brain were acquired on a 3T MRI scanner (Achieva, Philips Healthcare, Andover, MA) using a surface coil (RK-100 or LP-100; FUS instruments, Toronto, ON). At the time of the final PET session, group A animals weighed
$171.3 \pm 10.1 \mathrm{~g}$, group B animals weighed $243.4 \pm 12.9 \mathrm{~g}$, and group $C$ animals weighed $276.9 \pm 20.8$ g. Before each pFUS+MB session, each rat was infused with $100 \mu \mathrm{L}$ gadopentetate dimeglumine (Gd-DTPA, Magnevist ${ }^{\circ}$, Bayer Healthcare Pharmaceuticals, Inc., MA) via tail vein. Thirty seconds prior to initiating pFUS, an intravenous infusion of $100 \mu \mathrm{L}$ Optison $^{\mathrm{mm}}$ (GE Healthcare, Little Chalfont, Buckinghamshire, UK) (range 584$361 \mu \mathrm{L} / \mathrm{kg}$, at a rate of $1.66 \mu \mathrm{L} / \mathrm{s}$ ) was performed over $30 \mathrm{~s}$ as previously described [24,33]. Each animal in this study received the same number of Optison ${ }^{\text {Ts }} \mathrm{MB}(5-8 \times$ $10^{7}$ ) with an intravascular half-life of $0.72 \mathrm{~min}$ because animals were receiving $100 \%$ oxygen [24,33]. Sonication was targeted to the left frontal and right hippocampal regions with non-overlapping 2-mm-diameter focal regions and with a time lapse of $5 \mathrm{~min}$ between the two targeted regions. pFUS was performed using passive cavitation detection (PCD) in which the peak negative pressure (PNP) was changed in real time using proprietary hardware and software from the manufacturer (FUS Instruments, Toronto, ON) while monitoring the ultraharmonic frequencies at $1.5 f_{O}$ and $2.5 f_{o}$ to correct US pressures if the frequency exceeded 3.5 compared to fast Fourier transform (FFT) baseline [34]. The algorithm for PCD feedback included 10 US bursts at $0 \mathrm{~W}$ input power $(0 \mathrm{MPa})$ that were used as a baseline. The starting PNP for all groups was $0.144 \mathrm{MPa}$ with 
incremental increases of $0.008 \mathrm{MPa}$ with each pulse repetition frequency $(\mathrm{PRF}=0.5-0.6 \mathrm{~Hz}$ ). The pFUS parameters were as follows: transducer center frequency = $548 \mathrm{kHz}$ (FUS Instruments), focal diameter $=0.8$, active diameter $=7.5 \mathrm{~cm}$, burst $=10 \mathrm{~ms}$, and duty cycle $<1 \%$ with planned 100 sonications per focal spot over $120 \mathrm{~s}$. Axial T1-weighted images were acquired immediately after sonication to document the location and extent of gadolinium extravasation $(\mathrm{TR} / \mathrm{TE}=350 / 12 \mathrm{~ms})$.

In vivo T2\%-weighted MRI scans were performed on a Philips $3 \mathrm{~T}$ scanner (group A), Bruker 9.4T (Bruker Corp., Billerica, MA) (group B), and Bruker 7T (Bruker Corp., Billerica, MA) (group C) depending on the availability of the resources. For group A animals, axial T2*weighted images were acquired using a 3-cm-diameter solenoid coil (Philips Research Laboratories, Amsterdam,
Netherlands) and fast field echo (FFE) sequence with following parameters: $\mathrm{TR} / \mathrm{TE}=1301 / 7 \mathrm{~ms}, \Delta \mathrm{TE}=7 \mathrm{~ms}$, number of echoes $=5$, in-plane resolution $=100 \times$ $100 \mu \mathrm{m}^{2}$, and slice thickness $=500 \mu \mathrm{m}$. T2*-weighted images for group $\mathrm{B}$ were acquired using a multiple gradient echo (MGE) sequence with $\mathrm{TR} / \mathrm{TE}=50 / 3 \mathrm{~ms}$, $\Delta \mathrm{TE}=3 \mathrm{~ms}$, number of echoes $=10$, image resolution $=$ $200 \mu^{3}$ (isotropic) with a 5.0-cm Doty quadrature coil (Doty Scientific, Inc., Columbia, SC). For group C animals, $\mathrm{T} 2 \%$-weighted images were obtained using a $3.8-\mathrm{cm}$ Doty quadrature coil using MGE sequence with TR/TE = $60 / 2.4 \mathrm{~ms}, \Delta \mathrm{TE}=4.7 \mathrm{~ms}$, number of echoes $=10$, image resolution $=200 \times 200 \mu \mathrm{m}^{2}$, and slice thickness $=500 \mu \mathrm{m}$ (Fig. 2c). The final $\mathrm{T} 2{ }^{*}$-weighted images were generated by combining the multiple echo data to enhance the T2* contrast for the evaluation of abnormality.
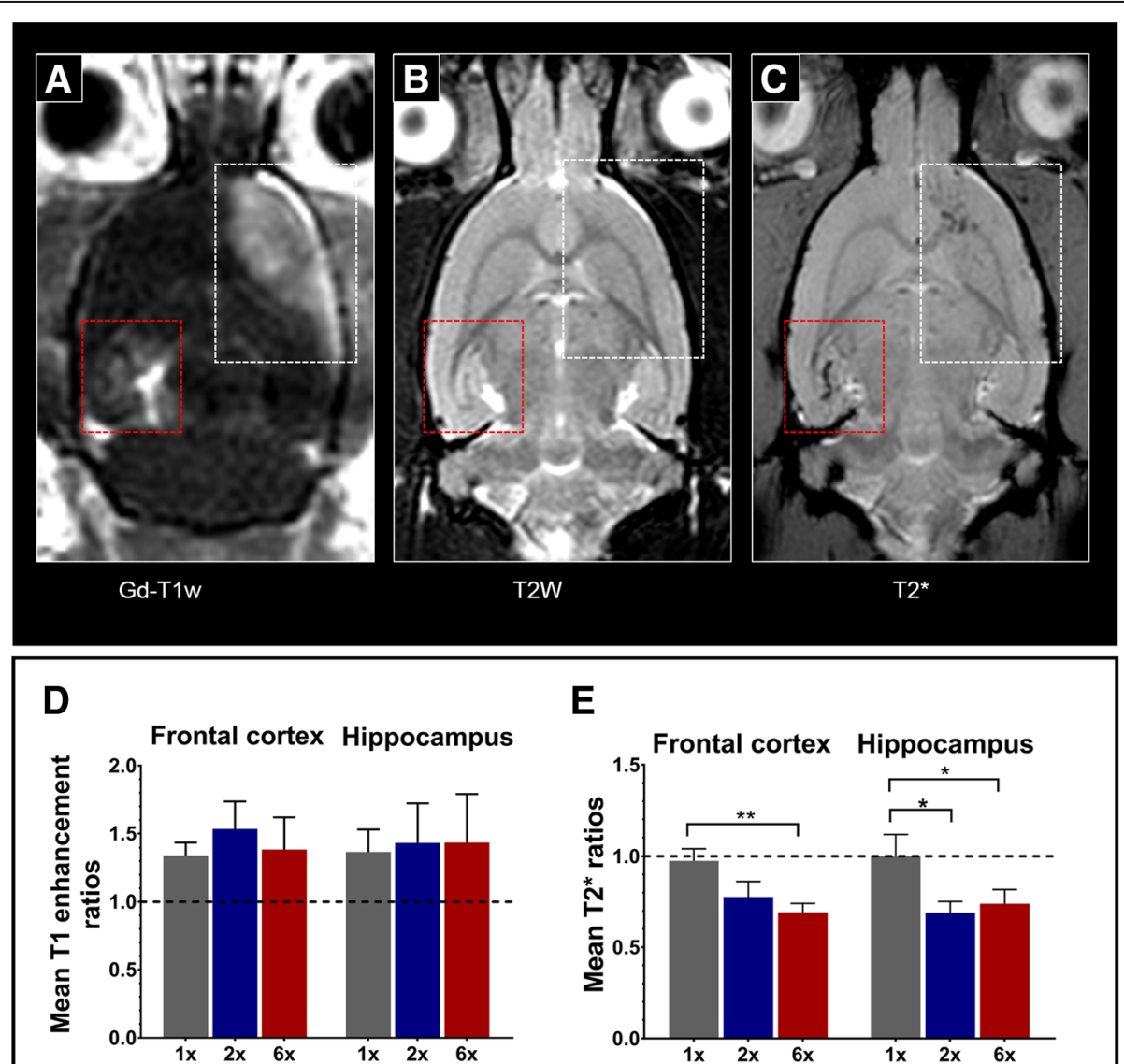

Fig. $2 \mathrm{MRI}$ findings in rats treated with pFUS+MB (a) T1-weighted scan obtained immediately following the first sonication showing contrast extravasation in the sonicated left frontal cortical (white rectangle) and right hippocampal region (red rectangle), reflecting efficient BBB disruption. b T2-weighted scan acquired after two sonications (week 3 ) showing no significant parenchymal signal abnormalities. c T2*-weighted scan obtained after six sonications (week 7) showing multiple hypointense foci in the sonicated regions, likely reflecting a combination of extravasated red blood cells, microhemorrhagic changes, and venous dilatation. $\mathbf{d}$ Mean ratios of T1 enhancement (sonicated/non-sonicated) in the three groups for one $(1 \times)$, two $(2 \times)$, and six $(6 \times)$ sonications. No statistically significant differences were detected (Kruskal-Wallis test, $p>0.05)$. e Mean T2* ratios (sonicated/non-sonicated) in the three groups showing significant incremental decrease in T2* ratios in the sonicated regions with increasing number of sonications (Kruskal-Wallis test, $p<0.05$, post-hoc Dunn's test: ${ }^{* *}$ denotes $p<0.005$ and ${ }^{*}$ denotes $p<0.05$ ). Error bars represent standard deviations 
In four animals from group A, high-resolution ex vivo MRI scans were performed following the imaging sessions, within 1 day after the sonication session. Following perfusion with $4 \%$ paraformaldehyde (PFA) in phosphate-buffered saline (PBS), the brains were extracted and immersed in perfluoropolyether fluorinated fluids (PFPE, Galden HS/260, Solvay Solexis, Brussels, Belgium) for scans. The ex vivo scans were performed on a Bruker $7 \mathrm{~T}$ scanner with T2-weighted RARE (TR/ $\mathrm{TE}=2747 / 15 \mathrm{~ms}$, RARE factor $=4$, in-plane resolution $=$ $78 \times 78 \mu \mathrm{m}^{2}$ with $500 \mu \mathrm{m}$ thickness) and T2*-weighted MGE $\quad(\mathrm{TR} / \mathrm{TE}=100 / 5 \mathrm{~ms}, \Delta \mathrm{TE}=5 \mathrm{~ms}$, number of echoes $=14$, flip angle $=30^{\circ}$, image resolution $=100 \mu \mathrm{m}^{3}$ isotropic).

In order to confirm reversal of the BBBO at the time of our earliest PET scan (24 h after sonication), five rats were imaged immediately after sonication (post contrast T1-weighted imaging) as well as $24 \mathrm{~h}$ after sonication (pre and post contrast T1-weighted images).

\section{[18F]DPA-714 radiosynthesis and PET imaging}

[18F]DPA-714 was synthesized as previously described [35]. The radiochemical yield was 40-60\% $(n>20)$; radiochemical purity was $>99 \%$; molar activity was 33$259 \mathrm{GBq} / \mu \mathrm{mol}$ at the end of synthesis. The concentration used for our experiments was $805 \pm 240 \mathrm{MBq} / \mathrm{mL}$ $(21.75 \pm 6.48 \mathrm{mCi} / \mathrm{mL})$ and molar activity during production was $116 \pm 64 \mathrm{GBq} / \mu \mathrm{mol}(3129 \pm 1737 \mathrm{Ci} / \mathrm{mmol})$.

For all PET imaging studies, rats were first anesthetized with $2-2.5 \%$ isoflurane-100\% oxygen mixture. Anesthesia levels were adjusted to maintain a target respiratory rate of 40-60 breaths/min. Body temperature was maintained by a heating pad.

A preclinical Inveon PET/CT scanner (Siemens Medical Solutions, USA) was used with following imaging parameters: transaxial and axial field of view (FOV) of 10 and $12.7 \mathrm{~cm}$, full width at half maximum spatial resolution at center FOV $=1.4 \mathrm{~mm}$. Two to three rats were scanned per imaging session. [18F]DPA-714 was injected through the tail vein (mean dose $=35.2 \mathrm{MBq}(0.951 \mathrm{mCi})$, mean mass dose at time of injection $=1.46 \pm 0.3 \mathrm{nmol} / \mathrm{kg}$ ) over a period of $30 \mathrm{~s}$ as a bolus followed by a quick saline flush $(300 \mu \mathrm{L})$. Thirty minutes after the injection of the radiotracer, the animal was secured to the scanner bed with its head placed symmetrically within the center FOV. Following CT acquisition for attenuation correction, PET emission scans were acquired in list mode starting at $40 \mathrm{~min}$ after injection. The choice for imaging time range is based on our previous experience with this ligand where we found that those frames (40-60 min) reflect "pseudoequilibrium" status since they had the lowest rate of change in the concentration activity curve $(<5 \% / \mathrm{h})$ [36]. The emission sinograms were corrected for scatter, ${ }^{18} \mathrm{~F}$ decay, random, and dead time. The resulting histograms were then reconstructed applying Fourier rebinning and 3D ordered subject expectation maximization algorithm (OSEM-3D; 4 OSEM iterations, 18 MAP iterations, matrix: $128 \times 128$, target resolution: $0.8 \mathrm{~mm}^{2}$ ). The animal was allowed to recover from anesthesia under a heat lamp after scan completion.

\section{MRI and PET image analysis}

Contrast enhancement on T1-weighted images was assessed immediately after the pFUS $+\mathrm{MB}$ in the sonicated frontal cortex and hippocampal region compared to the contralateral brain using ImageJ (NIH, Bethesda, USA). Regions of interest (ROIs) were first drawn in the sonicated regions and duplicated on the contralateral side. The ratios of enhancement in the sonicated regions compared to the contralateral brain were calculated using the mean image intensities.

Quantitative T2* maps were created by fitting the signal intensity (S) of each voxel from the MGE images to a mono-exponential decay as a function of TE: $S_{\mathrm{i}}=S_{0}$ exp. $\left(-\mathrm{TE}_{\mathrm{i}} / \mathrm{T} 2 *\right)$.

Volumes of interest (VOIs) encompassing sonicated and contralateral regions were drawn to extract the $\mathrm{T} 2 \%$ values. The $\mathrm{T}^{*}$ value ratios in the sonicated brain regions compared to the contralateral brain were then calculated from the T2* maps.

Analysis of the reconstructed PET images was done using PMOD 3.7 (PMOD technologies, Ltd., Zurich, Switzerland). First, PET images were co-registered to an MRI template. After co-registration, VOIs were drawn in the sonicated left frontal and right hippocampal regions and mirrored in the corresponding contralateral brain regions, using MR images from the sonicated animals for guidance. Control VOIs were drawn in both cerebellar hemispheres which were not sonicated. Care was taken to avoid including the choroid plexus within the lateral and fourth ventricles (known to have high TSPO expression [37]) and to avoid spillover radioactivity from extracranial structures with high uptake. Averaged percent injected dose/cc (\%ID/cc) were derived for these respective VOIs.

\section{Histological staining and analysis}

Rats $(n=11)$ including four from group A, three from group $B$, and four from group $C$ were euthanized immediately after the last PET imaging session. The animals were perfused with $4 \%$ paraformaldehyde fixative (PFA). The brains were then extracted and post-fixed in $4 \%$ PFA for 48 h. Fixed tissue blocks were embedded in paraffin wax blocks and sectioned at 3 or $5 \mu \mathrm{m}$ thickness. Axial brain sections (one section from each animal) including both the sonicated left frontal and right hippocampal regions were stained with Modified Mayer's Hematoxylin and Eosin-Y (H\&E). All slides were 
counterstained with DAPI (4',6-diamidino-2-phenylindole) at a concentration of $1 \mathrm{ng} / \mathrm{mL}$ to label cell nuclei. Primary antibodies used for IF: chicken anti-glial fibrillary acidic protein (GFAP) 1:200 (AB5541 Millipore Sigma, MA) and rabbit anti-ionized calcium binding adaptor molecule 1 (Iba1) 1:200 (019-19741 Wako Chemicals USA, VA).

Histological evaluation of the microscopy sections was performed at $\times 20$ magnification for all animals in each group. Aperio ScanScope CS equipped with a $\times 20$ air objective (NA $=0.75$, Leica Microsystems, Buffalo Grove, IL) was used for microscopy. One section from each brain was used for quantitative analysis. A laser scanning confocal microscope (model 710, Carl Zeiss AG, Oberkochen, Germany) using Plan-Apochromat objectives $(\times 20$ air, $\mathrm{NA}=0.8)$ was used for confocal microscopy. Illumination was provided by argon-ion (Lasos, Jena, Germany), diode and diode-pumped solid-state lasers (Roithner Lasertechnik, Vienna, Austria).

In each rat, ten FOVs were chosen in each of the sonicated regions and ten FOVs were chosen in the exact contralateral brain regions. Thresholds were selected to remove background signal, with the same threshold applied for all regions in the same animal. For each region, fluorescence signal was measured using Image and averaged from all the FOVs within that region. This process was performed sequentially for Iba1 and GFAP staining. Ipsilateral fluorescence signal was then compared to contralateral signal, giving a final value in the form of the ratio of sonicated to non-sonicated regions.

\section{Statistical analysis}

Ratio values of averaged \%ID/cc for [18F]DPA-714 PET binding is represented as mean \pm standard deviation (SD). Statistical analysis was performed using GraphPad Prism (version.7, GraphPad Software Inc.). For each sonicated region, T1-contrast enhancement ratios, $\mathrm{T} 2{ }^{*}$ value ratios, and $\% \mathrm{ID} / \mathrm{cc}$ ratios were first calculated. The ratios for staining (sonicated/non-sonicated) were also calculated for Iba1 and GFAP. Kruskal-Wallis non-parametric testing was then used to compare the three groups of animals for significant differences in T1-contrast enhancement, T2* values, and staining intensity. No comparison of PET binding among the three groups was performed due to acquisition of PET scans at different time points after the last sonication. When $p$ values were found to be $<0.05$, post-hoc Dunn's multiple comparison tests were performed.

For the animals that underwent repeated imaging after two and six weekly sonications $(n=3), \% \mathrm{ID} / \mathrm{cc}$ ratios were compared using paired $t$ test.
The significance threshold for all studies was set at $p<0.05$.

\section{Results \\ MRI findings}

Contrast enhancement on T1-weighted MRI immediately following pFUS $+\mathrm{MB}$ was identified in the sonicated regions for all three groups (Fig. 2a, Additional file 1: Figure S1). In five animals scanned $24 \mathrm{~h}$ after sonication, we did not identify any contrast enhancement confirming reversal of the BBBO at the time of our earliest PET scan (Additional file 1: Figure S1). Even though all animals showed definite enhancement in the ipsilateral sonicated brain compared to the contralateral side immediately after sonication, the ratios of T1-enhancement (sonicated/ non-sonicated) were not significantly different between the groups in either region $(p>0.05)$ (Fig. 2d). The ratios of $\mathrm{T}^{*}$ values (sonicated/non-sonicated) were significantly different between the three groups in the left frontal region $(p<0.0001)$ with post-hoc analysis showing significantly lower ratios in group $\mathrm{C}(6 \times)$ compared to group A $(1 \times)(p=0.0023)$. The ratios of $\mathrm{T}^{*}$ values were significantly different between groups in the right hippocampal region $(p=0.0018)$ with post-hoc analysis showing significantly lower ratios in group B $(2 \times)$ compared to group A $(1 \times)(p=0.02)$ and in group $C(6 \times)$ compared to group A $(p=0.039)$ (Fig. 2e).

\section{PET-imaging results}

In all groups, [18F]DPA-714 PET imaging showed mean $\% \mathrm{ID} / \mathrm{cc}$ ratios (sonicated/non-sonicated) that are $>1$ in the frontal cortical and hippocampal regions (Fig. 3, Tables 1 and 2). [18F]DPA-714 binding between the two cerebellar hemispheres was very similar for all three groups (ratio range of 0.98-1.03) (Table 1, Fig. 3c).

In a longitudinal analysis of three rats imaged after the second and then after the sixth sonication, the mean $\% \mathrm{ID} / \mathrm{cc}$ ratios were not significantly different in either region nor in the cerebellum $(p>0.05)$ (Fig. 3d).

\section{Histology findings in pFUS+MB-treated rats}

Increased GFAP and Iba1 staining related to pFUS+MB was noted in the sonicated regions compared to the nonsonicated (contralateral) brain in all three groups with representative examples shown in Fig. 4a. The averaged ratios (sonicated/non-sonicated fluorescent signal) were $>1$ in both regions; however, the changes were more impressive in the sonicated frontal cortex than in the sonicated hippocampus (compared to respective non-sonicated brains) (Fig. 4b, c). Those differences however did not reach significance in either location $(p>0.05)$. 

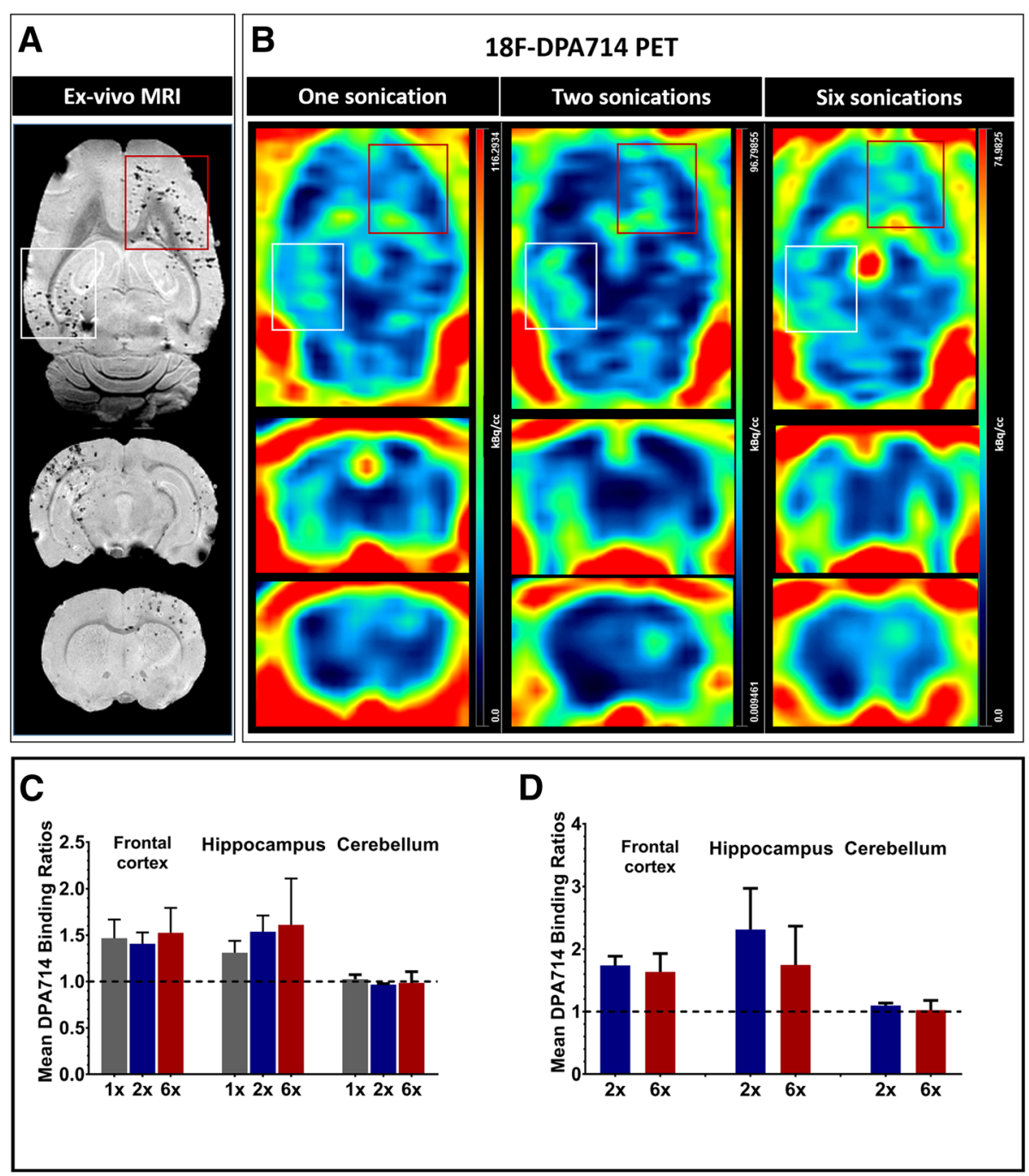

Fig. 3 PET imaging findings in rats treated with PFUS+MB (a) Ex vivo T2*-weighted MR images showing multiple hypointense foci in the sonicated frontal region (red box) and the right hippocampal region (white box) suggestive of extravasated red blood cells, microhemorrhagic changes and hemosiderin within phagocytic cells. b Three examples of [18F]DPA-714 PET scans showing increased binding in the sonicated regions after one, two, and six sonication sessions respectively. c Ratios of [18F]DPA-714 binding (sonicated/non-sonicated mean \%ID/cc values) in both sonicated regions following one (1X), two (2X), and six (6X) sonications. No increased [18F]DPA-714 seen in the cerebellar hemispheres which were not sonicated. $\mathbf{d}$ Longitudinal mean ratios of [18F]DPA-714 binding in a subset of animals after two $(2 x)$ and six $(6 \times)$ sonications. No statistically significant differences were detected in the sonicated regions or in the cerebellum (control region) (Paired t-test, $p>0.05$ ). Error bars represent standard deviations

Table 1 Mean ratio of [18F]DPA-714 binding (sonicated/nonsonicated) in the frontal cortex and hippocampus in all three groups of animals. The cerebellum was not sonicated and is used as control

\begin{tabular}{llll}
\hline $\begin{array}{l}\text { Region of } \\
\text { interest }\end{array}$ & \multicolumn{3}{l}{ Mean ratio of ipsilateral/contralateral } \\
\cline { 2 - 4 } & $\begin{array}{l}\text { 1 sonication } \\
N=6\end{array}$ & $\begin{array}{l}2 \text { sonications } \\
N=8\end{array}$ & $\begin{array}{l}6 \text { sonications } \\
N=5\end{array}$ \\
\hline Frontal cortex & $1.47 \pm 0.2$ & $1.53 \pm 0.2$ & $1.53 \pm 0.3$ \\
Hippocampus & $1.31 \pm 0.1$ & $1.83 \pm 0.5$ & $1.61 \pm 0.5$ \\
Cerebellum & $1.03 \pm 0.04$ & $1.02 \pm 0.07$ & $0.98 \pm 0.12$ \\
\hline
\end{tabular}

Table 2 Mean ratio of [18F]DPA-714 binding (sonicated/nonsonicated) in the frontal cortex and hippocampus in animals that were followed longitudinally after two and six sonications. The cerebellum was not sonicated and is used as control

\begin{tabular}{lll}
\hline Region of & \multicolumn{2}{l}{ Mean ratio of ipsilateral/contralateral } \\
\cline { 2 - 3 } interest & 2 sonications & 6 sonications \\
\hline Frontal cortex & $1.74 \pm 0.1$ & $1.64 \pm 0.3$ \\
Hippocampus & $2.31 \pm 0.7$ & $1.74 \pm 0.6$ \\
Cerebellum & $1.10 \pm 0.04$ & $1.02 \pm 0.1$ \\
\hline
\end{tabular}



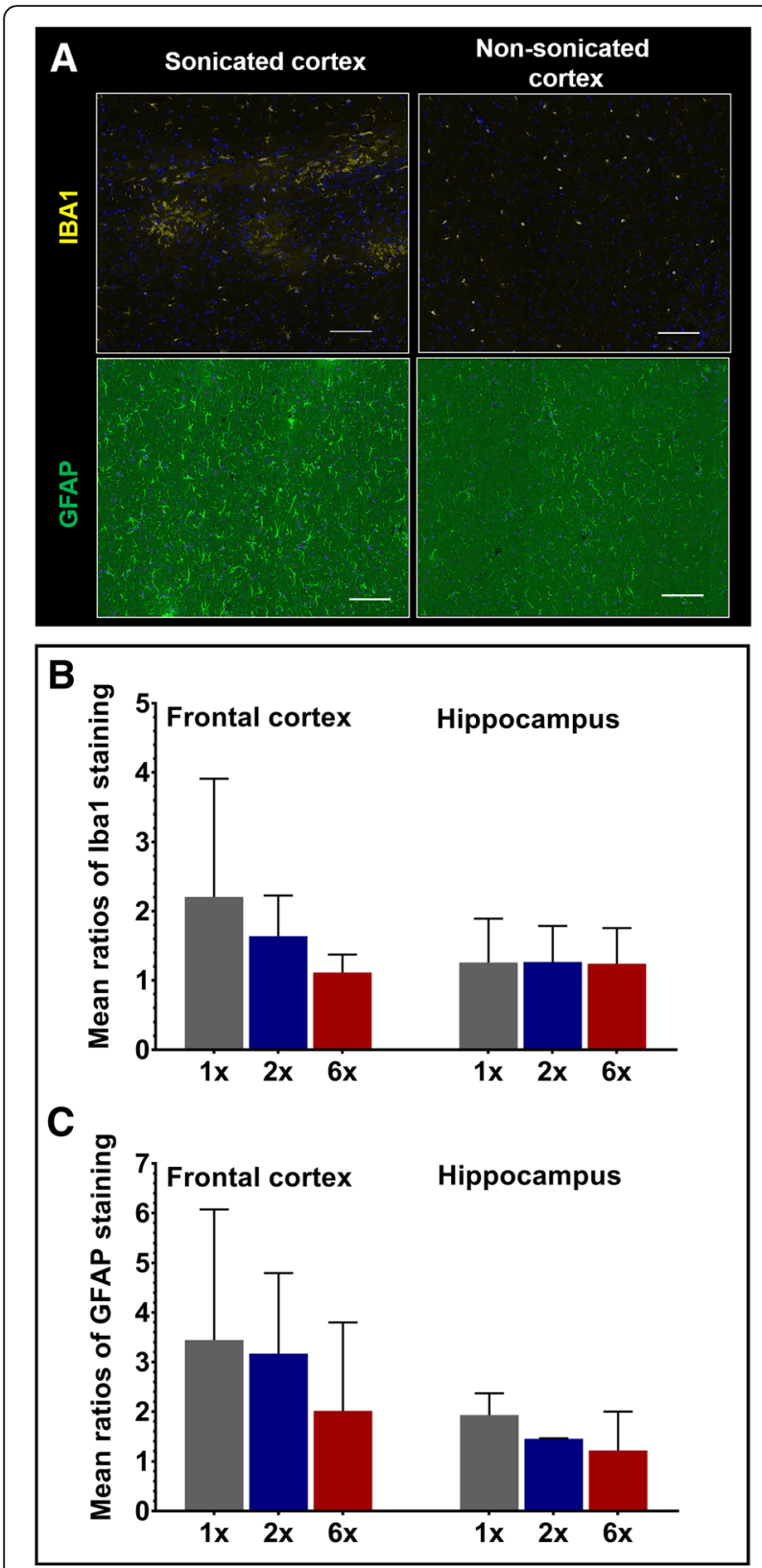

Fig. 4 Histopathologic correlates in pFUS+MB treated rats. a Ibal and GFAP staining from sonicated and contralateral non-sonicated brain (frontal cortex) showing patchy foci of increased Ibal staining as well as more diffuse increase in GFAP staining. $\mathbf{b}$ Mean Iba1 and $\mathbf{c}$ mean GFAP fluorescence signal ratios (sonicated/non-sonicated) in the frontal and hippocampal regions respectively. Error bars represent standard deviations

\section{Discussion}

MRIgFUS with MB is a proposed approach to open the $\mathrm{BBB}$ for purposes of drug delivery in specific targeted brain regions $[8-12,38,39]$. The mechanism of $\mathrm{BBBO}$ by pFUS $+\mathrm{MB}$ is likely due to acoustic radiation pressure generated by ultrasound along with acoustic stable or inertial cavitation effects from the intravascular $\mathrm{MB}$ oscillations. Secondary stretching of the endothelial cells coupled with induced expression of cytokines, chemokines, and trophic factors (CCTF) from the various cellular components of the NVU and alterations in tight junction integrity eventually result in BBBO $[14,40,41]$. pFUS+MB-induced transient BBBO has already been used for neurotherapeutics' delivery in experimental models of disease [42-44] and has been proposed to increase beta-amyloid $(A \beta)$ clearance from the brains of AD mouse models [45-47]. More recently, serial pFUS+MB has been used in various clinical trials to open the $\mathrm{BBB}$ (clinicaltrials.gov: NCT02986932, NCT03119961, NCT03347084), mainly as a method to decrease $A \beta$ deposition in patients with $A D$ [20], and in patients with CNS malignancy [21]. Despite the purported benefits of pFUS+MB, side effects of this technique, such as potential neuroinflammatory sequelae, have received little attention.

Recent studies have suggested that $\mathrm{pFUS}+\mathrm{MB}$ can induce a sterile inflammatory response (SIR) as evidenced by increased proteomic and transcriptomic expression of proinflammatory CCTF [14, 24, 33]. In our study, we were able to visualize those neuroinflammatory changes using noninvasive [18F]DPA-714 PET imaging. Our results were not confounded by leakage across a disrupted BBB since by the time we imaged the animals $(24 \mathrm{~h}$ following first sonication and 1-2 weeks after the second and sixth sonications), the acute BBB disruption by pFUS would have completely resolved [48, 49]. In fact, we confirmed reversal of $\mathrm{BBBO}$ in a separate group of five animals by documenting lack of contrast leakage on MRI in the sonicated regions $24 \mathrm{~h}$ after sonication (Additional file 1: Figure S1). Our PET imaging findings were further supported by increased Ibal and GFAP immunofluorescent staining in the sonicated areas consistent with microglial activation and astrocytosis. The increased [18F]DPA-714 binding in the sonication sites was observed as early as $24 \mathrm{~h}$ after one sonication (Fig. 3a, b) as well as for days to weeks after two and six weekly pFUS+MB exposures (Fig. 3c). We did not compare binding between the three groups due to differences in time lapses between PET scanning and the last sonication. However, in the longitudinal cohort of animals that was imaged within a comparable time frame after the second and sixth sonications, we did not see significant differences in [18F]DPA-714 binding (Fig. 3d, Table 2) suggesting no PET-detectable evidence of $\mathrm{cu}$ mulative inflammatory effect. Our findings support the notion of an SIR that persists for at least 2 weeks after the reversal of $\mathrm{BBB}$ disruption, although we have no PET evidence of additive effect of multiple sonications based on a small subset of animals imaged longitudinally.

Using T1-weighted imaging, we confirmed successful BBBO immediately post pFUS+MB (Fig. 2a). The degree 
of enhancement seen immediately post sonication did not significantly change between groups (Fig. 2d) which is not surprising since $\mathrm{BBBO}$ is expected to have reversed within the time interval between the sessions [14, 49]. This was confirmed by lack of contrast enhancement in a separate group of animals imaged with MRI $24 \mathrm{~h}$ after sonication. There was however an incremental further decrease in $\mathrm{T} 2$ * values after two and six sonications reaching statistical significance in both sonicated regions when compared to one sonication (Fig. 2e). As previously described, those findings reflect microhemorrhagic changes related to vascular injury and microglia and macrophages (metallophagocytic cells) phagocytosing the red blood cells in the parenchyma. Secondary iron deposition ( $\mathrm{T} 2 *$ signal) is detected and increases as a function of the number of sonication sessions $[14,24]$. Some of the hypointense voxels also reflect slow blood flow in dilated vessels as previously demonstrated [24]. Of note, hypointense voxels on $3 \mathrm{~T} \mathrm{~T} 2{ }^{*}$-weighted images were recently reported in two out of five $\mathrm{AD}$ patients who received pFUS+MB to open the BBB [20].

There have been multiple discussions in the literature related to the comparability of pFUS $+\mathrm{MB}$ parameters/ dose between the various preclinical studies and their equivalency to human applications. The picture however remains complex with multiple factors affecting the endresult of efficient BBB disruption [14, 18, 24, 33, 50, 51]. In practical terms, the magnitude of $\mathrm{BBBO}$ following pFUS+MB is dependent upon multiple parameters including the MB type (size and sonographic characteristics), infusion rate (bolus vs. slow infusion), dose, and initial concentration of injected MB as well as other experimental conditions including oxygenation levels, PNP, and PCD parameters $[18,33,50-53]$. To add to the complexity, there are several approaches for PCD feedback used to limit MB cavitation [54-57], although it is not clear which is the optimal method for controlling PNP changes that would limit parenchymal injury. In our study, we used $100 \%$ oxygenation which significantly decreases the half-life of $\mathrm{MB}$ in the vasculature (0.72 min compared to $1.43 \mathrm{~min}$ at $21 \%$ oxygenation) [58-60]. We also used PCD feedback of PNP in order to limit the magnitude of ultraharmonics at $1.5 f o$ and $2.5 f o$ to less than 3.5 and minimize parenchymal injury by limiting the amount of stable cavitation from intravascular MB [55]. We used Optison at $100 \mu \mathrm{L}\left(5-8 \times 10^{7} \mathrm{MB} /\right.$ rat) independent of weight, infused slowly over $1 \mathrm{~min}$ with sonication delayed by $30 \mathrm{~s}$ allowing for the $\mathrm{MB}$ concentration in the vasculature to reach near steadystate [33]. As the animals increased in age and weight, the effective $\mathrm{MB}$ concentration administered to groups $\mathrm{B}$ and $C$ decreased as a consequence of increasing weight. Moreover, a recent study has shown that repeated exposures to Optison will significantly shorten the intravascular half-life of the MB [61], which would further decrease the effective dose of $\mathrm{MB}$ used in group $\mathrm{B}$ and $\mathrm{C}$ rats compared to group A rats.

Although it is difficult to compare our procedural parameters to those used in humans [20], the MB dose (total \#MB) used in the current study is within the range of preclinical experimental protocol parameters reported in the literature to cause $\mathrm{BBBO}$ following pFUS with or without PCD feedback [24, 54, 62, 63]. Most relevant to many of the clinical trials targeting AD-afflicted subjects, our experimental pFUS parameters are comparable to those used in animal studies showing the effectiveness of FUS in clearance of $A \beta$ plaques in mouse models $[45-47,64,65]$. Theoretically, if the clinical protocols using lower concentrations of $\mathrm{MB}$ do not achieve a similar level of BBBO as those in the preclinical studies, the effectiveness and human translatability of the approach would be in jeopardy. Limitations associated with human TSPO imaging such as the known polymorphism of the TSPO gene (rs6971) resulting in different binding affinities, should be taken into account; quantification however is simplified considering the focal nature of $\mathrm{pFUS}+\mathrm{MB}$ allowing the use of contralateral non-sonicated brain as reference region without the extra hassle of arterial blood sampling [26].

As we have previously mentioned [33], the main questions regarding the utility of pFUS in human clinical trials can be summarized as follows: (1) What pFUS parameters are needed to induce the necessary BBB disruption for adequate parenchymal delivery of neurotherapeutics or stimulation of the required immune response? (2) What risks would the combination of those sonication parameters impose as far as inducing a neuroinflammatory reaction with potential secondary tissue damage? Using the current method with parameters that are comparable to those used in most preclinical studies [24], we have shown an SIR in animals undergoing MRI-guided pFUS+MB opening of the BBB in vivo, using PET imaging, as early as $24 \mathrm{~h}$ after single sonication. Multiple sonications resulted in worsening of the MRI findings especially hemosiderin deposition. However, there was no statistically significant increase in the associated SIR, neither by imaging nor by histology. This could suggest resolution of the inflammatory changes in-between sessions.

\section{Conclusion}

Considering the great clinical potential of pFUS+MB, our findings warrant a deeper exploration of the optimal experimental parameters in human clinical trials needed to induce a therapeutically useful degree of BBBO but without the potentially harmful neuroinflammatory effects. [18F]DPA-714 PET along with MRI could be used to determine those optimal pFUS+MB parameters which would minimize the negative effects of the intervention while achieving the necessary BBBO. 


\section{Additional file}

Additional file 1: Figure S1. MRI scans obtained immediately after sonication show contrast leakage in the left frontal region (red box) and the right hippocampal region (white box). Repeat imaging after $24 \mathrm{~h}$ shows resolution of contrast leakage consistent with reversal of BBB opening. (TIF $784 \mathrm{~kb}$ )

\section{Abbreviations}

\%ID/cc: Percent injected dose/cc; AD: Alzheimer's disease; BBB: Blood-brain barrier; BBBO: Blood-brain barrier opening; CCTF: Cytokines, chemokines, and trophic factors; DAMP: Damage-associated molecular pattern; FFE: Fast field echo; FFT: Fast Fourier transform; FOV: Field of view; GFAP: Glial fibrillary acidic protein; Iba1: Ionized calcium binding adaptor molecule 1; MB: Microbubbles; MGE: Multiple gradient echo; MRI: Magnetic resonance imaging; NVU: Neurovascular unit; OSEM: Ordered subject expectation maximization algorithm; PBS: Phosphate-buffered saline; PCD: Passive cavitation detection feedback; PFA: Paraformaldehyde;

PFPE: Perfluoropolyether fluorinated fluids; pFUS+MB: Pulsed focused ultrasound + microbubbles; PNP: Peak negative pressure; PRF: Pulse repetition frequency; ROI: Region of interest; SIR: Sterile inflammatory response; TR/TE: Repetition time/echo time; TSPO: Translocator protein; VOI: Volume of interest

\section{Acknowledgements}

Not applicable.

\section{Authors' contributions}

$\mathrm{DAH}$ and JAF conceived and planned the study, contributed to data collection and analysis, and finalized the manuscript. SS, TT, ZIK, WS-S, MS, GZP, and WCR performed experiments, collected and analyzed the corresponding data. SS and DAH performed the statistical analysis. SS prepared the first version of the manuscript including tables and graphs. All authors reviewed and approved the manuscript.

\section{Funding}

This work was supported by the Intramural Research Programs of the CC and $\mathrm{NIBIB}$ at the NIH. Dr. Hammoud and Dr. Frank's lab equally supported this research. Dr. Tsang-Wei Tu was funded by the Department of Defense through the CNRM (Henry M. Jackson Foundation Award \#308049-10.01-60855, USU Site No. CNRM-89-3899).

\section{Availability of data and materials}

All reported data can be made available to qualified investigators upon request.

\section{Ethics approval}

All procedures were approved by the Animal Care and Use Committee of the Clinical Center, National Institute of Health $(\mathrm{NIH})$.

\section{Consent for publication}

Not applicable.

\section{Competing interests}

The authors declare that they have no competing interests.

\footnotetext{
Author details

${ }^{1}$ Hammoud Laboratory, Center for Infectious Disease Imaging, Clinical Center, National Institutes of Health, 10 Center Drive, Building 10, Room 1C-368, Bethesda, MD 20892, USA. Frank Laboratory, Radiology and Imaging Sciences, Clinical Center, National Institutes of Health, Bethesda, MD, USA. ${ }^{3}$ Center for Neuroscience and Regenerative Medicine, Uniformed Services University of the Health Sciences, Bethesda, MD, USA. ${ }^{4}$ Imaging Probe Development Center, National Heart, Lung, and Blood Institute, National Institutes of Health, Rockville, MD, USA. ${ }^{5}$ National Institute of Biomedical Imaging and Bioengineering, National Institutes of Health, Bethesda, MD, USA. ${ }^{6}$ University of Texas, MD Anderson Cancer Center, Houston, USA. ${ }^{7}$ Department of Radiology, Howard University, Washington DC, USA. ${ }^{8}$ Institute for Biomedical Engineering, Swiss Federal Institute of Technology,
}

Zurich, Switzerland. ${ }^{9}$ Department of Radiology, University of Crete and Department of Medical Imaging Heraklion University Hospital, Crete, Greece.

Received: 27 March 2019 Accepted: 9 July 2019

Published online: 25 July 2019

\section{References}

1. McConnell HL, Kersch CN, Woltjer RL, Neuwelt EA. The translational significance of the neurovascular unit. J Biol Chem. 2017;292(3):762-70.

2. Zhao Z, Nelson AR, Betsholtz C, Zlokovic BV. Establishment and dysfunction of the blood-brain barrier. Cell. 2015;163(5):1064-78.

3. Banks WA. From blood-brain barrier to blood-brain interface: new opportunities for CNS drug delivery. Nat Rev Drug Discov. 2016;15(4):275-92.

4. Suzuki Y, Nagai N, Umemura K. A review of the mechanisms of blood-brain barrier permeability by tissue-type plasminogen activator treatment for cerebral ischemia. Front Cell Neurosci. 2016;10:2.

5. Pardridge WM. Drug and gene delivery to the brain: the vascular route. Neuron. 2002;36(4):555-8.

6. Gomez D, Martinez JA, Hanson LR, Frey WH 2nd, Toth CC. Intranasal treatment of neurodegenerative diseases and stroke. Front Biosci (Schol Ed). 2012:4:74-89.

7. Neuwelt E, Abbott NJ, Abrey L, Banks WA, Blakley B, Davis T, et al. Strategies to advance translational research into brain barriers. Lancet Neurol. 2008; 7(1):84-96.

8. Nhan T, Burgess A, Cho EE, Stefanovic B, Lilge L, Hynynen K. Drug delivery to the brain by focused ultrasound induced blood-brain barrier disruption: quantitative evaluation of enhanced permeability of cerebral vasculature using two-photon microscopy. J Control Release. 2013;172(1):274-80.

9. Tempany CMC, McDannold NJ, Hynynen K, Jolesz FA. Focused ultrasound surgery in oncology: overview and principles. Radiology. 2011;259(1):39-56.

10. Sheikov N, McDannold N, Sharma S, Hynynen K. Effect of focused ultrasound applied with an ultrasound contrast agent on the tight junctional integrity of the brain microvascular endothelium. Ultrasound Med Biol. 2008;34(7):1093-104

11. McDannold N, Vykhodtseva N, Hynynen K. Effects of acoustic parameters and ultrasound contrast agent dose on focused-ultrasound induced blood-brain barrier disruption. Ultrasound Med Biol. 2008;34(6):930-7.

12. Hynynen K, McDannold N, Vykhodtseva N, Jolesz FA. Noninvasive MR imaging-guided focal opening of the blood-brain barrier in rabbits. Radiology. 2001;220(3):640-6.

13. Goertz DE. An overview of the influence of therapeutic ultrasound exposures on the vasculature: high intensity ultrasound and microbubblemediated bioeffects. Int J Hyperth. 2015;31(2):134-44

14. Kovacs Zl, Kim S, Jikaria N, Qureshi F, Milo B, Lewis BK, et al. Disrupting the blood-brain barrier by focused ultrasound induces sterile inflammation. Proc Natl Acad Sci. 2017;114(1):E75-84.

15. Chen GY, Nunez G. Sterile inflammation: sensing and reacting to damage. Nat Rev Immunol. 2010;10(12):826-37.

16. Gadani SP, Walsh JT, Lukens JR, Kipnis J. Dealing with danger in the CNS: the response of the immune system to injury. Neuron. 2015;87(1):47-62.

17. Tuttolomondo A, Pecoraro R, Pinto A. Studies of selective TNF inhibitors in the treatment of brain injury from stroke and trauma: a review of the evidence to date. Drug Des Devel Ther. 2014:8:2221-38.

18. McMahon D, Hynynen K. Acute inflammatory response following increased blood-brain barrier permeability induced by focused ultrasound is dependent on microbubble dose. Theranostics. 2017;7(16):3989-4000.

19. Burgess A, Hynynen K. Drug delivery across the blood-brain barrier using focused ultrasound. Expert Opin Drug Deliv. 2014;11(5):711-21.

20. Lipsman $N$, Meng $Y$, Bethune AJ, Huang $Y$, Lam B, Masellis $M$, et al. Bloodbrain barrier opening in Alzheimer's disease using MR-guided focused ultrasound. Nat Commun. 2018;9(1):2336.

21. Mainprize $T$, Lipsman N, Huang $Y$, Meng $Y$, Bethune A, Ironside $S$, et al. Blood-brain barrier opening in primary brain tumors with non-invasive MRguided focused ultrasound: a clinical safety and feasibility study. Sci Rep. 2019;9(1):321

22. O'Reilly MA, Jones RM, Barrett E, Schwab A, Head E, Hynynen K. Investigation of the safety of focused ultrasound-induced blood-brain barrier opening in a natural canine model of aging. Theranostics. 2017:7(14): 3573-84.

23. Chu PC, Chai WY, Tsai CH, Kang ST, Yeh CK, Liu HL. Focused ultrasound-induced blood-brain barrier opening: association with mechanical index and cavitation 
index analyzed by dynamic contrast-enhanced magnetic-resonance imaging. Sci Rep. 2016;6:33264.

24. Kovacs ZI, Tu TW, Sundby M, Qureshi F, Lewis BK, Jikaria N, et al. MRI and histological evaluation of pulsed focused ultrasound and microbubbles treatment effects in the brain. Theranostics. 2018;8(17):4837-55.

25. Venneti S, Lopresti BJ, Wiley CA. The peripheral benzodiazepine receptor (translocator protein 18kDa) in microglia: from pathology to imaging. Prog Neurobiol. 2006;80(6):308-22.

26. Hammoud DA. Molecular imaging of inflammation: current status. J Nucl Med. 2016;57(8):1161-5.

27. Harhausen D, Sudmann V, Khojasteh U, Muller J, Zille M, Graham K, et al. Specific imaging of inflammation with the $18 \mathrm{kDa}$ translocator protein ligand DPA-714 in animal models of epilepsy and stroke. PLoS One. 2013;8(8):e69529.

28. Awde AR, Boisgard R, Theze B, Dubois A, Zheng J, Dolle F, et al. The translocator protein radioligand $18 \mathrm{~F}-\mathrm{DPA}-714$ monitors antitumor effect of erufosine in a rat $9 \mathrm{~L}$ intracranial glioma model. J Nucl Med. 2013;54(12):2125-31.

29. Martin A, Boisgard R, Kassiou M, Dolle F, Tavitian B. Reduced PBR/TSPO expression after minocycline treatment in a rat model of focal cerebral ischemia: a PET study using [(18) F]DPA-714. Mol Imaging Biol. 2011;13(1):10-5

30. Golla SS, Boellaard R, Oikonen V, Hoffmann A, van Berckel BN, Windhorst $A D$, et al. Quantification of [18F]DPA-714 binding in the human brain: initial studies in healthy controls and Alzheimer's disease patients. J Cereb Blood Flow Metab. 2015;35(5):766-72.

31. Ribeiro MJ, Vercouillie J, Debiais S, Cottier JP, Bonnaud I, Camus V, et al. Could (18) F-DPA-714 PET imaging be interesting to use in the early poststroke period? EJNMMI Res. 2014;4:28.

32. Committee for the Update of the Guide for the Care and Use of Laboratory Animals. Guide for the care and use of laboratory animals, 8th edition, (2011).

33. Kovacs ZI, Burks SR, Frank JA. Focused ultrasound with microbubbles induces sterile inflammatory response proportional to the blood brain barrier opening: attention to experimental conditions. Theranostics. 2018;8(8):2245-8.

34. O'Reilly MA, Hough O, Hynynen K. Blood-brain barrier closure time after controlled ultrasound-induced opening is independent of opening volume. J Ultrasound Med. 2017;36(3):475-83.

35. Kuhnast B, Damont A, Hinnen F, Catarina T, Demphel S, Le Helleix S, et al. [18F]DPA-714, [18F]PBR111 and [18F]FEDAA1106 — selective radioligands for imaging TSPO 18kDa with PET: automated radiosynthesis on a TRACERLAb FX-FN synthesizer and quality controls. Appl Radiat Isot. 2012;70(3):489-97.

36. Lee $D E$, Yue $X$, Ibrahim WG, Lentz MR, Peterson KL, Jagoda EM, et al. Lack of neuroinflammation in the HIV-1 transgenic rat: an [(18) F]-DPA714 PET imaging study. J Neuroinflammation. 2015;12:171.

37. Benavides J, Cornu P, Dennis T, Dubois A, Hauw JJ, Mackenzie ET, et al. Imaging of human brain lesions with an omega 3 site radioligand. Ann Neurol. 1988;24(6):708-12.

38. Raymond SB, Treat LH, Dewey JD, McDannold NJ, Hynynen K, Bacskai BJ. Ultrasound enhanced delivery of molecular imaging and therapeutic agents in Alzheimer's disease mouse models. PLoS One. 2008:3(5):e2175.

39. Vykhodtseva N, McDannold N, Hynynen K. Progress and problems in the application of focused ultrasound for blood-brain barrier disruption. Ultrasonics. 2008;48(4):279-96.

40. Borden MA, Kruse DE, Caskey CF, Zhao S, Dayton PA, Ferrara KW. Influence of lipid shell physicochemical properties on ultrasound-induced microbubble destruction. IEEE Trans Ultrason Ferroelectr Freq Control. 2005;52(11):1992-2002.

41. James JC, Kirsten S, Zimeng G, Gesthimani S, Babak B, Elisa EK. Noninvasive and localized blood-brain barrier disruption using focused ultrasound can be achieved at short pulse lengths and low pulse repetition frequencies. J Cereb Blood Flow Metab. 2010;31(2):725-37.

42. Horodyckid C, Canney M, Vignot A, Boisgard R, Drier A, Huberfeld G, et al. Safe long-term repeated disruption of the blood-brain barrier using an implantable ultrasound device: a multiparametric study in a primate model. J Neurosurg. 2017;126(4):1351-61.

43. Downs ME, Buch A, Karakatsani ME, Konofagou EE, Ferrera VP. Blood-brain barrier opening in behaving non-human primates via focused ultrasound with systemically administered microbubbles. Sci Rep. 2015;5:15076.

44. Olumolade OO, Wang S, Samiotaki G, Konofagou EE. Longitudinal motor and behavioral assessment of blood-brain barrier opening with transcranial focused ultrasound. Ultrasound Med Biol. 2016;42(9):2270-82.

45. Burgess A, Dubey S, Yeung S, Hough O, Eterman N, Aubert I, et al. Alzheimer disease in a mouse model: MR imaging-guided focused ultrasound targeted to the hippocampus opens the blood-brain barrier and improves pathologic abnormalities and behavior. Radiology. 2014;273(3):736-45.
46. Jordao JF, Thevenot E, Markham-Coultes K, Scarcelli T, Weng YQ, Xhima K, et al. Amyloid-beta plaque reduction, endogenous antibody delivery and glial activation by brain-targeted, transcranial focused ultrasound. Exp Neurol. 2013;248:16-29.

47. Leinenga G, Gotz J. Scanning ultrasound removes amyloid-beta and restores memory in an Alzheimer's disease mouse model. Sci Transl Med. 2015;7(278): $278 \mathrm{ra33}$.

48. Alonso A, Reinz E, Jenne JW, Fatar M, Schmidt-Glenewinkel H, Hennerici MG, et al. Reorganization of gap junctions after focused ultrasound blood-brain barrier opening in the rat brain. J Cereb Blood Flow Metab. 2010;30(7): 1394-402.

49. Samiotaki G, Konofagou EE. Dependence of the reversibility of focused- ultrasoundinduced blood-brain barrier opening on pressure and pulse length in vivo. IEEE Trans Ultrason Ferroelectr Freq Control. 2013;60(11):2257-65.

50. Silburt J, Lipsman N, Aubert I. Disrupting the blood-brain barrier with focused ultrasound: perspectives on inflammation and regeneration. Proc Natl Acad Sci. 2017;114(33):E6735-E6.

51. McMahon D, Hynynen K. Reply to Kovacs et al.: concerning acute inflammatory response following focused ultrasound and microbubbles in the brain. Theranostics. 2018;8(8):2249-50.

52. Zsofia I, Kovacs SRB, Joseph A. Frank. Focused ultrasound with microbubbles induces sterile inflammatory response proportional to the blood brain barrier opening: attention to experimental conditions. Theranostics. 2018;8(8):2245-8.

53. Kovacs ZI, Burks SR, Frank JA. Reply to Silburt et al.: concerning sterile inflammation following focused ultrasound and microbubbles in the brain. Proc Natl Acad Sci. 2017;114(33):E6737-E8.

54. Kamimura HA, Flament J, Valette J, Cafarelli A, Aron Badin R, Hantraye P, et al. Feedback control of microbubble cavitation for ultrasound-mediated blood-brain barrier disruption in non-human primates under magnetic resonance guidance. $J$ Cereb Blood Flow Metab. 2018; https://doi.org/10.1177/0271678X17753514.

55. O'Reilly MA, Hynynen K. Blood-brain barrier: real-time feedback-controlled focused ultrasound disruption by using an acoustic emissions-based controller. Radiology. 2012;263(1):96-106.

56. Sun T, Zhang Y, Power C, Alexander PM, Sutton JT, Aryal M, et al. Closedloop control of targeted ultrasound drug delivery across the blood-brain/ tumor barriers in a rat glioma model. Proc Natl Acad Sci U S A. 2017; 114(48):E10281-e90.

57. Tung YS, Vlachos F, Choi JJ, Deffieux T, Selert K, Konofagou EE. In vivo transcranial cavitation threshold detection during ultrasound-induced blood-brain barrier opening in mice. Phys Med Biol. 2010;55(20):6141-55.

58. Itani M, Mattrey RF. The effect of inhaled gases on ultrasound contrast agent longevity in vivo. Mol Imaging Biol. 2012;14(1):40-6.

59. McDannold N, Zhang Y, Vykhodtseva N. The effects of oxygen on ultrasound-induced blood-brain barrier disruption in mice. Ultrasound Med Biol. 2017;43(2):469-75.

60. Mullin L, Gessner R, Kwan J, Kaya M, Borden MA, Dayton PA. Effect of anesthesia carrier gas on in vivo circulation times of ultrasound microbubble contrast agents in rats. Contrast Media Mol Imaging. 2011;6(3):126-31.

61. Fix SM, Nyankima AG, McSweeney MD, Tsuruta JK, Lai SK, Dayton PA. Accelerated clearance of ultrasound contrast agents containing polyethylene glycol is associated with the generation of anti-polyethylene glycol antibodies. Ultrasound Med Biol. 2018;44(6):1266-80.

62. Bing C, Hong Y, Hernandez C, Rich M, Cheng B, Munaweera I, et al. Characterization of different bubble formulations for blood-brain barrier opening using a focused ultrasound system with acoustic feedback control. Sci Rep. 2018:8(1):7986

63. Burgess MT, Apostolakis I, Konofagou EE. Power cavitation-guided bloodbrain barrier opening with focused ultrasound and microbubbles. Phys Med Biol. 2018;63(6):065009.

64. Hsu PH, Lin YT, Chung YH, Lin KJ, Yang LY, Yen TC, et al. Focused ultrasound-induced blood-brain barrier opening enhances GSK-3 inhibitor delivery for amyloid-Beta plaque reduction. Sci Rep. 2018;8(1):12882.

65. Poon CT, Shah K, Lin C, Tse R, Kim KK, Mooney S, et al. Time course of focused ultrasound effects on beta-amyloid plaque pathology in the TgCRND8 mouse model of Alzheimer's disease. Sci Rep. 2018;8(1):14061.

\section{Publisher's Note}

Springer Nature remains neutral with regard to jurisdictional claims in published maps and institutional affiliations. 